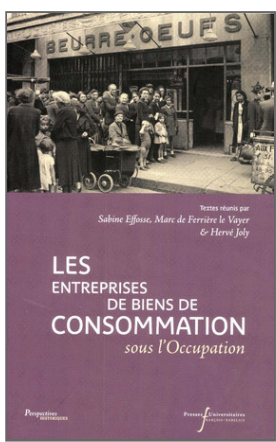

Sabine Effosse, Marc de Ferrière Le Vayer et Hervé Joly (dir.)

Les entreprises de biens de consommation sous

l'Occupation

Presses universitaires François-Rabelais

\title{
Entre espoirs et déceptions : l'industrie des pâtes alimentaires
}

Pierre-Antoine Dessaux

DOI : 10.4000/books.pufr. 1894

Éditeur : Presses universitaires François-Rabelais

Lieu d'édition : Presses universitaires François-Rabelais

Année d'édition : 2010

Date de mise en ligne : 22 mai 2013

Collection : Perspectives Historiques

EAN électronique : 9782869063327

\section{Doentation}

http://books.openedition.org

Ce document vous est offert par Université de Tours

\section{U}

\section{Référence électronique}

DESSAUX, Pierre-Antoine. Entre espoirs et déceptions : l'industrie des pâtes alimentaires In : Les entreprises de biens de consommation sous l'Occupation [en ligne]. Tours : Presses universitaires François-Rabelais, 2010 (généré le 22 octobre 2021). Disponible sur Internet : <http://

books.openedition.org/pufr/1894>. ISBN : 9782869063327. DOI : https://doi.org/10.4000/books.pufr. 1894.

Ce document a été généré automatiquement le 22 octobre 2021. Il est issu d'une numérisation par reconnaissance optique de caractères. 


\title{
Entre espoirs et déceptions : l'industrie des pâtes alimentaires
}

\author{
Pierre-Antoine Dessaux
}

1 LA PÉRIODE de l'Occupation fut relativement favorable à l'industrie des pâtes alimentaires. Inscrites au ravitaillement civil et militaire depuis la Grande Guerre, susceptibles d'être produites à partir de matières premières nationales et surtout reconnues autant pour leurs qualités nutritives que pour leur faculté à assurer une forme de stockage des céréales capable d'améliorer la gestion de la soudure, les pâtes alimentaires bénéficièrent de toute évidence d'un régime de faveur en matière d'allocation des contingents de matières premières. La production distribuée s'accrut de près de $25 \%$ sur la période ce qui engendra, au même titre que la Première Guerre mondiale, une rupture durable, à la hausse, de la tendance de la consommation moyenne.

2 Pour les principaux industriels de l'époque, pour l'essentiel basés à Lyon et disposant de plusieurs établissements sur le territoire, le bilan de la période n'en fut pas moins mitigé. Confrontés à de sérieuses difficultés commerciales durant les années 1930, certains virent dans l'organisation corporative et ses promesses de rationalisation de la production un espoir d'amélioration de leurs positions et de leur rentabilité. Les difficultés structurelles de la période en décidèrent autrement et l'industrie, prioritairement soumise à des enjeux de ravitaillement local, en sortit plus fragmentée qu'elle n'y était entrée. Pour autant, l'organisation professionnelle qui fut alors mise en place connut une destinée singulière, puisque le comité professionnel de la branche et les outils de contingentement et de contrôle furent maintenus bien au-delà de la période de pénurie. Ils servirent en effet aussi bien les intérêts de l'État que de certains industriels ${ }^{1}$. 


\section{Une organisation collective favorable aux industriels dominants}

3 Bien que de sensibilité plutôt libérale, les principaux industriels de la branche pâtes alimentaires accueillirent plutôt favorablement l'organisation économique promue au sein de la révolution nationale. Ils avaient en effet pu constater certains des avantages de l'encadrement corporatif du marché des céréales mis en place durant les années 1930. L'extension de ces logiques à leurs propres activités pouvait être perçue comme une solution aux difficultés rencontrées durant l'entre-deux-guerres.

Industrie née au XIX ${ }^{e}$ siècle et ayant atteint une certaine maturité au début du Xxe siècle, la fabrique de pâtes alimentaires traversa l'entre-deux-guerres entre des espoirs de croissance déçus et la prédominance des questions de commercialisation. En inscrivant les pâtes alimentaires au rang des denrées rationnées, les autorités du ravitaillement de la Grande Guerre avaient contribué à relancer la croissance de la demande, qui tripla durant le conflit, ce qui assura un niveau de profit considérable mais entraîna la fragmentation des structures industrielles $d u$ fait de l'apparition de nombreux nouveaux producteurs. Une fois passée l'inflation des années 1920, l'industrie fut donc marquée par une vive concurrence et une dégradation de ses résultats.

5 L'organisation du marché du blé mise en place dans les années 1930 apporta un peu de répit. La réglementation des qualités de matières premières à employer puis, en 1934, l'obligation de recours aux productions de l'Empire contribuèrent à restreindre le champ de la concurrence par les qualités de produit. La mise en place, en 1936, de l'office national interprofessionnel du blé (ONIB) vint à son tour éliminer le potentiel de concurrence fondé sur les stratégies d'approvisionnement. A partir de 1938, les productions de semoules de blé dur, essentiellement destinées à la fabrication des pâtes alimentaires, firent l'objet d'une garantie de revenu minimum au moyen d'un système de contingentement de l'activité. Les coûts d'approvisionnement en matière première furent donc stabilisés. Comme ils représentaient une part essentielle des coûts de production, les perspectives d'une concurrence tarifaire s'en trouvèrent largement restreintes. A la veille des hostilités, les industriels pouvaient être plutôt favorables à un encadrement du marché susceptible de garantir la consolidation de leurs bénéfices.

6 L'expérience du ravitaillement mis en place en 1916 avait de surcroît laissé des traces plutôt positives dans les entreprises qui étaient alors déjà actives. La pénurie étant prévisible et les modalités de mise en place du ravitaillement étant largement laissées aux mains des industriels, ces derniers purent constituer des stocks et jouer sur l'augmentation mécanique des prix pour dégager de conséquents bénéfices. Les premiers mois de la drôle de guerre puis de l'occupation permirent de renouveler l'expérience. Prévu dès septembre 1939, le rationnement des pâtes alimentaires ne fut, au nom du moral, pas immédiatement mis en place. Les besoins militaires et les réflexes de stockage engendrèrent donc une augmentation conséquente des ventes qui soutint une croissance de $21 \%$ de la production tandis que les prix s'envolaient de plus de $50 \%^{2}$. Cela suffit à faire sortir l'industrie du marasme qui l'avait caractérisée durant les années 1930. Rivoire \& Carret, la première entreprise de la branche, améliora ses bénéfices de $127 \%$ en francs constants pour une production qui n'augmenta que de $85 \%$. Et ses concurrents ne furent pas en reste. Les liquidités ainsi dégagées furent largement employées à la constitution de stocks considérables, à caractère essentiellement spéculatif, même si ils étaient en partie destinés à assurer la continuité 
de la production. Établi en juin 1940, le bilan du principal établissement marseillais de Rivoire \& Carret fait ainsi apparaître un quintuplement en francs constants du poste matières premières par rapport à l'année précédente, le portant 11,6 millions de francs. La même pratique se retrouve à Grenoble chez Cartier-Millon, la maison de la marque Lustucru. Elle fut probablement d'autant plus générale que l'expérience passée du ravitaillement avait également démontré l'importance des stocks pour justifier de volumes de production à partir desquels les éventuels contingentements à venir pouvaient être négociés ${ }^{3}$.

7 L'organisation professionnelle voulue par le nouveau régime à partir de la loi sur les comités d'organisation du 16 août 1940 fut d'abord bien accueillie par les principaux industriels. En s'affichant comme corporative, elle semblait promettre le recul du contrôle public propre aux groupements d'achat et de répartition des denrées alimentaires. Chez Rivoire \& Carret, dont les dirigeants affichaient les valeurs antiétatiques traditionnelles du patronat lyonnais, l'adhésion fut d'abord clairement affichée et résultait autant d'une certaine résignation aux nécessités du moment que de l'espoir de contrôler cette nouvelle organisation :

La création, au sein de chaque Profession, d'un «Comité professionnel » dans lequel entrera un Commissaire du Gouvernement, représente d'ailleurs l'acheminement non pas vers un Étatisme absolu et à sens unique, menace de naguère, mais vers la formation d'un ensemble dont les bases seront constituées par les organisations syndicales et professionnelles.

Par le Syndicalisme et le Professionnalisme Provinciaux, le régionalisme renaîtra.

Si dans cette vaste Révolution les initiatives Privées ne seront plus à même d'évoluer aussi librement qu'autrefois, les milieux représentatifs de chaque profession auront indiscutablement leur mot à dire et ils seront d'autant mieux écoutés qu'ils auront un programme constructif et seront l'expression d'un bloc cohésif ${ }^{4}$.

8 La disparition des organisations nationales et le repli sur une gestion corporative censée être dépolitisée laissaient entrevoir une maitrise directe du cadre professionnel à laquelle nombre d'industriels souscrirent de bonne volonté.

9 Le premier objectif collectif porta sur l'autonomisation des approvisionnements. Avec le tarissement des importations de blés durs en provenance d'Afrique du Nord, la filière pâtes alimentaires se trouvait en concurrence directe avec les autres filières céréalières, dominantes au sein de l'office national interprofessionnel des céréales (ONIC), pour la répartition des ressources intérieures. Parce que la production de pâtes pouvait permettre de mieux gérer le calendrier des consommations de céréales, le ravitaillement concéda la mise à disposition très en amont de matières premières. Un Groupement des industriels de la semoulerie et des pâtes alimentaires (GISPA) fut par ailleurs créé pour assurer la collecte et la répartition des matières premières. Dès la fin 1940, l'horizon d'activité pouvait sembler relativement assuré. Il était possible d'envisager une politique de branche.

10 La nouvelle organisation corporative et sa soumission aux impératifs du ravitaillement présentaient l'indéniable avantage de résoudre le problème commercial caractéristique de l'entre-deux-guerres. La répartition autoritaire des contingents auprès des détaillants suspendait la concurrence et permettait de réduire à néant l'effort commercial qui avait précédemment atteint des niveaux considérables du fait de la concurrence. Pour les industriels dominants, qui avaient largement peiné à améliorer leur rentabilité durant les années 1930, la corporation et le contingentement 
représentaient une opportunité majeure pour consolider leurs débouchés et asseoir une position durable via l'élimination de la concurrence. Dans la filière, l'industrie semoulière avait déjà fait l'objet, dans le cadre de l'ONIB, d'une politique de restructuration assise sur la gestion des contingentements. Il ne s'agissait que d'étendre cette démarche à la seconde transformation.

11 L'organisation corporative de l'industrie des pâtes alimentaires définie par la loi du 24 juin $1941^{5}$ qui instaure le comité professionnel de l'industrie des pâtes alimentaires (CPIPA) se fixa donc dès l'origine des objectifs de rationalisation qui répondaient directement aux vœux de principaux industriels. Ses missions portèrent évidemment sur les prix, la répartition des matières premières, la statistique professionnelle et l'instruction des dossiers de demande d'ouverture ou d'extension des établissements, mais également sur la modernisation sectorielle orientée vers «la résorption progressive et méthodique des moyens de production et des contingents de fabrications par leur rachat corporatif et volontaire ». Son objectif officiel était de réduire les capacités de production aux «besoins de la consommation nationale et de l'exportation moyenne des trois dernières années augmentée de $50 \%$ ». La concurrence n'était pas totalement éliminée sur le papier, mais les capacités des principales entreprises auraient largement suffi à remplir cet objectif. La loi traduisait donc certains des intérêts de la branche que l'on retrouve sans surprise dans les nominations au comité professionnel. L'arrêté d'installation du 4 août $1941^{6}$ désigna ainsi sans surprise les plus ardents partisans d'une limitation de la concurrence aux fins de soutenir des politiques de marque et de prix imposés. Nommé au poste stratégique de responsable des calculs de contingents, Jean Carret, actionnaire principal et président en exercice de Rivoire \& Carret, y occupa par exemple une place de premier plan.

Les six premiers mois d'activité du comité furent justement occupés par l'élaboration des règles de contingentement. La question était cruciale puisqu'elle déterminait directement l'activité des entreprises en période de pénurie mais risquait également de figer à long terme leurs parts de marché. Elle allait également fixer la base des éventuels transferts qui devaient déboucher sur la réduction du nombre d'établissements. Des contingents devaient également découler l'évaluation des coûts de production moyens sur la base desquels pouvait être fixée la marge industrielle qui rémunérait les producteurs. Il n'était donc pas nécessairement intéressant que les établissements les plus efficaces disposent de l'essentiel des volumes. Les négociations professionnelles aboutirent à une règle relativement ésotérique entérinée par un arrêté interministériel du 31 janvier $1942^{7}$. Le contingent annuel de base de chaque entreprise devait ainsi être égal à deux fois le nombre de quintaux mis en œuvre sur trois mois consécutifs choisis durant une période comprise entre le $1^{\mathrm{er}}$ août 1931 et le 3 juin 1941 et du nombre de quintaux livrés à la consommation durant une période de six mois consécutifs durant une période comprise entre le $\mathrm{I}^{\mathrm{er}}$ septembre 1929 et le 31 août 1939. Les établissements créés au lendemain de la déclaration de guerre étaient eux soumis à une évaluation nettement plus réduite et corrigée d'un coefficient exprimant les surcapacités de production de la branche, ce qui permettait de réduire largement leurs droits aux attributions. Au contingent initial pouvait enfin être ajoutée une marge de croissance de $10 \%$ en cas d'anticipation de la croissance de la consommation. Le but de la règle de contingentement était de prendre l'activité et non les capacités de production pour référence, ce qui ne correspondait pas directement à un objectif de rationalisation des équipements mais bien à celui de réduction de la concurrence : les 
entreprises les plus actives commercialement devaient être privilégiées indépendamment des besoins locaux ou de l'état réel de l'outil de production.

Dans la mesure où les statistiques de l'activité étaient encore relativement incertaines, les industriels étaient fortement incités à gonfler une production mal connue. Certains, comme Rivoire \& Carret, n'hésitèrent pas à augmenter leurs références, tandis que la règle de contingentement permettait malgré les critiques de prendre en considération l'activité exceptionnelle de l'année 1940. L'entreprise lyonnaise obtint ainsi des contingents largement supérieurs à sa production d'avant-guerre. D'un trait de plume, elle qui bénéficiait, comme le soulignaient ses administrateurs, "des relations personnellement entretenues par Monsieur Jean Carret avec le ministre de l'Agriculture et du Ravitaillement ${ }^{8}$ " put ainsi se retrouver en position extrêmement forte sur son marché. Les comptes de la société furent également manifestement maquillés pour masquer des surestimations sur les années de référence. Au total, les quatre premières sociétés de la branche s'arrogeaient $44 \%$ des contingents : 13,62 \% pour Ferrand \& Renaud à Lyon, 12,79\% pour le groupe Rivoire \& Carret SIPA Duniel, $10,4 \%$ pour Bozon-Verduraz à Grenoble et $6,87 \%$ pour Pâtes alimentaires de France également à Lyon. Les dix premières sociétés revendiquaient près de $60 \%$ des contingents. Une répartition (concentration) sur le papier probablement largement artificielle mais qui ouvrait la voie à une politique ultérieure de concentration industrielle.

14 L'organisation initiale de la corporation favorisait donc manifestement la poursuite des objectifs de rentabilisation par la réduction de la concurrence prônée par les industriels les plus influents. Sa mise en œuvre fut cependant soumise aux contraintes du moment et, bien que bénéficiant d'un soutien à l'activité, échoua totalement à restructurer la branche.

\section{Une trajectoire d'entreprise dominante}

15 À l'instar de ce qui a pu être observé dans de nombreuses autres entreprises dominantes dans leur branche d'activité et par conséquent intimement associées à la mise en place des organisations corporatives, la direction de Rivoire \& Carret adhéra tout d'abord largement aux orientations du régime. Bien que celles-ci heurtaient largement des convictions anti-interventionnistes jusque-là âprement défendues, elles laissaient entrevoir une piste en faveur de la réduction de la concurrence et de la consolidation de la position de marché. Les difficultés du ravitaillement vinrent cependant assez rapidement les remettre en cause.

16 La première phase du conflit fut plutôt positive. En novembre 1940, Jean Carret estimait que: "les événements nous ont permis d'opérer de sérieux redressements, des modifications que seules des situations exceptionnelles peuvent permettre ${ }^{9}$ ». L'activité avait en effet considérablement augmenté. Les chiffres de tonnage sont sujets à caution en raison des maquillages destinés à la négociation des contingents mais la rentabilité, qui était négative à la veille du conflit en raison d'investissements commerciaux peu efficaces, retrouva un niveau tout à fait conséquent pour l'époque $(3,9 \%)$ grâce à l'écoulement des stocks et à l'augmentation du prix de vente moyen. L'entreprise put ainsi disposer d'un important volume de liquidités qui lui permit pour partie de faire face aux retards de paiement qui apparurent avec le déclenchement des hostilités. Ces derniers représentèrent jusqu'à 6 millions de francs, soit près de $10 \%$ des ventes dans 
le courant de l'année 1940, mais ils n'empêchèrent pas de constituer d'importants stocks de matières premières. C'est donc à partir d'une position économique solide que Rivoire \& Carret, dont les activités industrielles étaient essentiellement implantées en zone non occupée, put envisager les perspectives de l'organisation corporative.

L'année 1941 fut encore relativement favorable. La mise en place effective du ravitaillement contribua à réduire considérablement les frais commerciaux, à commencer par la publicité, ce qui confortait la rentabilité de l'entreprise. Mais Rivoire \& Carret n'en supprima pas pour autant son service commercial difficilement reconstitué dans les années 1930 après une tentative de stratégie de "vente automatique " axée sur la publicité menée dans les années 1920. Dans l'espoir d'un retour rapide à des conditions d'activité normales, les personnels commerciaux furent maintenus. Ils furent de fait rémunérés jusqu'à la fin de la période, autant sans doute au motif d'une certaine responsabilité sociale que parce que l'entreprise connaissait parfaitement le coût d'une reconstitution des liens commerciaux. Le maintien d'une flotte de véhicules et de dépenses de déplacement suggère également que, malgré le caractère bureaucratique de l'allocation des produits, les commerciaux maintinrent une certaine activité de visite de leur clientèle. Il s'agissait d'éviter que d'autres occupent la place et surtout de maintenir le caractère opérationnel des structures commerciales dans l'attente d'un retour à la normale.

18 L'année 1942 marque là aussi, sans surprises, un tournant. Bien que Rivoire \& Carret ait obtenu des contingents tout à fait conséquents, les contraintes du ravitaillement se firent plus pressantes. La direction commença à formuler des critiques plus directes. L'économie dirigée, que l'on espérait être une approche pragmatique, se révélait être une improvisation "peu cohérente » aux tendances manifestement étatistes. Jean Carret se mit à déplorer qu'» en somme, nous travaillons pratiquement pour l'Etat qui devient de plus en plus l'actionnaire le mieux servi ». Des bénéfices n'en sont pas moins dégagés et la stratégie de vente sous marque put être maintenue grâce à l'obtention de matières premières pour les emballages négociées en contrepartie de fournitures aux troupes occupantes. Malgré les difficultés, il n'y avait pas lieu de remettre en cause les orientations durables de l'entreprise: "Malgré la tempête, il ne faut jamais se laisser démoraliser, il faut continuer notre marche en avant. En vertu du principe qu'à l'époque que nous vivons, «toute affaire qui ne se développe pas est une affaire qui se meurt », «il faut donc nous développer ${ }^{10} »$, pouvait-il encore souligner. L'essentiel restait d'occuper les positions en vue du retour à la normale.

19 La position de la direction à l'égard du régime commença cependant à basculer dans le courant de l'hiver 1942-1943. Il devenait en effet parfaitement clair que le tournant de l'économie dirigée n'était plus compatible avec la poursuite des objectifs de l'entreprise. Lors des conseils d'administration tenus en juin 1943, il fut décidé de dupliquer les rapports d'activité pour réserver à une série confidentielle le sentiment profond que la direction désirait destiner à l'actionnariat familial. S'y expriment sans détour les désillusions de l'évolution du régime et de son approche de l'industrie. Les fonctionnaires du ravitaillement y sont qualifiés de «bande d'incasables d'avant la guerre, d'ambitieux et d'incompétents ». L'obligation, prise en décembre 1942, de maintenir les matières premières dans les zones où leur transformation était possible invalide par ailleurs tout espoir de concentration industrielle comme de consolidation du marché au moyen de la politique corporative de contingentement. Avec le renforcement des tensions sur le ravitaillement, la tutelle de l'Etat se fit plus pressante. 
Dès lors, force était de constater que «si quelque système apparaitt comme éloigné des techniques corporatives, c'est bien celui qui attribue la direction de la Profession à des hommes désignés par voie d'autorité et, en outre, étroitement subordonnés aux représentants des pouvoirs publics ${ }^{11}$ ». Après avoir œuvré à la mise en place des institutions corporatives, Jean Carret réorienta son action vers la remobilisation d'une organisation professionnelle indépendante destinée à préparer des jours meilleurs. Son entreprise n'en parvenait pas moins à maintenir des résultats honorables. Alors que le Service du travail obligatoire (STO) mobilisait désormais $13 \%$ de la main-d'œuvre masculine, la rente associée à un régime de prix toujours favorable aux entreprises qui, comme la sienne, étaient les plus productives, permettait de maintenir le volume des bénéfices.

De la mi-1943 à mi-1944, Rivoire \& Carret entra manifestement en résistance vis-à-vis de ses autorités de tutelle. Il s'agissait de contrer ces «fonctionnaires, qui cherchent à coiffer la Profession [et] ne sont pas encore parvenus à leurs fins ». Dans les rapports de force, la manipulation des informations devint plus systématique, mais elle ne put cependant éviter la dégradation des conditions d'activité. Au final, l'ensemble de la période de l'Occupation paraît plutôt favorable à une entreprise comme Rivoire \& Carret. Précocement engagée dans l'action corporative, elle chercha à y promouvoir ses principaux intérêts et parvint à les inscrire dans les objectifs collectifs. Ses derniers ne furent que très partiellement atteints. L'essentiel des résultats tint au maintien de l'activité et de sa rentabilité, malgré l'accroissement des difficultés. L'emploi put globalement être protégé et les personnels soutenus par une indéniable solidarité interne qui passa là aussi par le développement d'activités agricoles annexes destinées au ravitaillement. Les actionnaires familiaux ne furent pas en reste. L'entreprise put maintenir le versement de confortables dividendes.

21 Les principales difficultés allaient plutôt venir des années de reconstruction. La stratégie globale mise en œuvre au travers des institutions corporatives et de la gestion du ravitaillement fut en effet plutôt un échec. Le ravitaillement avait finalement imposé une décentralisation de la production plutôt qu'une concentration. La priorité avait été donnée à la sécurisation des approvisionnements locaux et à la limitation des transports, toujours hasardeux, de marchandises.

Les grandes entreprises comme Rivoire \& Carret durent faire face à la fragmentation d'un marché qu'elles commençaient tout juste à unifier et sur lequel s'étaient de fait implantés de nouveaux concurrents orientés vers des marchés très localisés. En contradiction totale avec les objectifs corporatistes, les années 1940 furent marquées par l'émiettement de la structure de production.

\section{L'apparition de nouveaux acteurs $^{12}$}

Les objectifs de restructuration industrielle prônés par la corporation se heurtèrent rapidement aux nécessités du ravitaillement. L'expérience de la Première Guerre mondiale avait déjà montré l'importance d'une décentralisation au niveau départemental en vue de limiter la circulation des denrées et d'ajuster au mieux les ressources locales aux besoins locaux. Les transferts de matières premières et de marchandises ne pouvaient être justifiés que par les inégalités territoriales qui impliquaient une allocation prise en charge par les institutions centrales. Or le découpage du territoire issu de la convention d'armistice compliquait d'emblée cette 
gestion et les ponctions de l'occupant invitèrent rapidement à limiter au maximum ces transferts. Ces derniers étaient cependant tout particulièrement nécessaires dans la mesure où l'essentiel de l'outil industriel de l'industrie des pâtes alimentaires se trouvait en zone non occupée tandis que les trois-quarts des ressources en grains se situaient au nord de la ligne de démarcation. Les disponibilités en céréales du Sud de la France pouvaient plus ou moins permettre de faire fonctionner l'outil industriel lyonnais et marseillais, mais la coupure du territoire créait un appel d'air pour le développement et la création d'établissements de production dans la zone occupée.

De 1940 à 1945, le nombre d'établissements de production augmenta d'au moins soixante-dix unités, soit de près d'un quart. Vingt-quatre départements qui n'avaient aucune tradition en la matière virent apparaître cette activité. Elle émergea pour l'essentiel aux marges des zones de production dans une très grande couronne parisienne et à l'est de la vallée du Rhône, ainsi que dans l'Ouest, lorsque les disponibilités de matières premières le permettaient. Dans ces départements, les préfectures appuyèrent la création de nouveaux établissements, au besoin en contradiction avec les orientations du comité professionnel, afin d'assurer l'approvisionnement des populations locales et de maintenir sur zone les disponibilités en céréales. Au sortir de la guerre, la fabrication des pâtes alimentaires, qui était auparavant relativement concentrée dans les régions parisienne, lyonnaise et marseillaise, tendait à prendre une configuration d'industrie de proximité. La production était désormais assurée dans 64 départements contre 44 avant-guerre.

L'essentiel de ces nouveaux établissements étaient de dimensions restreintes, le plus souvent artisanales, mais certains purent, comme celui fondé par Jean Panzani, trouver sur la période les moyens d'un premier développement. Né à Paris en 1911, dans une famille de petite bourgeoisie industrielle et commerciale fraîchement immigrée d'Italie, Jean Panzani achevait sa formation d'expert-comptable auprès de la Compagnie industrielle des pétroles lors de la mobilisation. Démobilisé à l'automne 1940, il dut rejoindre sa famille à Niort (Deux-Sèvres) et trouver, comme beaucoup, une nouvelle activité. L'épargne du salaire maintenu durant la mobilisation et surtout une expérience familiale dans le commerce de produits alimentaires et la fabrication de pâtes le conduisirent à tenter cette activité qui, au moins dans sa forme artisanale, ne requiert pratiquement pas d'équipements. Les premières fabrications eurent lieu avec quelques kilos de farine, dans un grenier, et avec l'assistance familiale puis de deux apprentis. Elles étaient écoulées dans deux boulangeries qui assurèrent, avant que le ravitaillement ne se resserre, la mise à disposition des matières premières.

L'antériorité du démarrage de l'activité sur les mesures de contrôle des approvisionnements et de rationnement des produits permit d'obtenir une certaine légitimité pour poursuivre. Aucune entreprise ne produisait de pâtes alimentaires dans les Deux-Sèvres avant 1940, mais la préfecture appuya la création de deux autres établissements qui permettaient d'assurer la mise à disposition d'une denrée attendue par les consommateurs du seul fait de sa présence sur les cartes de ravitaillement. Lorsque la production de pâtes fraîches fut interdite en mars 1941, Jean Panzani assurait une production équivalente à 3000 rations mensuelles ${ }^{13}$. Le passage aux nouvelles normes de production, orientées vers la seule fabrication de pâtes sèches, requérait de véritables équipements. Là encore, la préfecture appuya la reconnaissance de la jeune entreprise par le CPIPA afin qu'elle obtienne les matériels adéquats. Des relations personnelles avec la maison parisienne Piscaglia qui importait avant-guerre 
des équipements italiens et s'était convertie à leur fabrication pour maintenir son activité permirent de disposer des équipements les plus récents, financés sur crédit fournisseur. La petite entreprise niortaise disposa donc dès fin 1941 de la première presse automatique continue de la profession. L'entreprise se développa à mesure que la pression sur les matières premières incitait les autorités locales à en conserver la plus grande part dans ce département producteur. À la Libération, l'établissement employait une cinquantaine de personnes. Une petite activité industrielle relativement solide et bien outillée avait donc pu se consolider en trois ans, grâce à l'élargissement de la palette des productions, qui employaient des farines de légumes disponibles localement, mais aussi des contingents spécifiques obtenus pour assurer le ravitaillement des troupes alliées dès la Libération; l'activité avait donc pu non seulement se maintenir mais aussi croitre en dépit des difficultés. Les conditions de démarrage étaient en outre objectivement favorables du fait de la pénurie et de l'absence totale de concurrence. En dépit de l'absence d'activité véritablement commerciale, l'entreprise disposait néanmoins, à la fin de la période, d'une certaine emprise sur le marché local et put alors espérer en tirer la base d'un développement ultérieur.

\section{Un marché qui évolue}

L'organisation du ravitaillement de pâtes alimentaires et les efforts consentis pour maintenir un certain niveau de production contribuèrent, comme après 1916, à modifier sensiblement le profil de la consommation. Alors que la moyenne annuelle ne dépassait pas 3,5 $\mathrm{kg}$ d'après les statistiques professionnelles de 1938, les autorités du ravitaillement tablèrent sur une consommation théorique de $5 \mathrm{~kg}$ par tête en moyenne $\mathrm{e}^{14}$. Un tel choix reflétait la promotion que les milieux de l'alimentation rationnelle faisaient des pâtes alimentaires depuis près de trente ans. Leur forte valeur nutritive était régulièrement soulignée. Leur production au titre d'emploi alternatif des farines permettait en outre de soustraire une partie des disponibilités à la production de pain dont on souhaitait ouvertement réduire la consommation. Leurs qualités de conservation en faisaient enfin une denrée idéale pour gérer les fluctuations saisonnières comme les aléas de la production agricole.

Alors que la consommation de pâtes alimentaires se partageait encore largement entre un emploi en soupes et potages et un usage en gratin, les rations allouées par le ravitaillement visèrent explicitement à en faire, au cœur de l'hiver, un produit de substitution aux pommes de terres. Les pâtes qui étaient encore jusque-là plutôt considérées comme un produit farineux furent plus systématiquement classées parmi les féculents et employées en conséquence comme un légume dans la structure du repas. Cet emploi avait déjà été promu durant la Grande Guerre, mais le ravitaillement touchait désormais vraiment l'ensemble de la population, à commencer par les zones, en particulier de l'Ouest, où ces produits étaient très peu consommés, et où de nouvelles habitudes purent apparaître. Il en résulta également une saisonnalité de l'activité nettement plus marquée.

29 En dépit d'une politique du ravitaillement très favorable à sa croissance, la production ne permit pas d'atteindre les objectifs affichés de consommation par tête. La production officielle s'établit ainsi autour de 200000 tonnes en 1942-1943 comme en 1945-1946, un volume qui représentait en tout état de cause une progression générale 
mais impliquait une géographie différenciée des mises à disposition et par suite de la consommation. L'effort de ravitaillement des villes se fit ainsi d'autant plus au détriment des campagnes que l'essentiel de l'appareil de production était situé en zone urbaine. Le consommateur rural, très diversement intéressé par le produit, fut celui dont les rations furent au final les plus écornées. Une étude menée en 1949 par le ministère des Finances soulignait un renforcement de la consommation à Paris et une baisse marquée aussi bien dans le Sud-Est, traditionnellement producteur et consommateur, que dans les zones rurales. Globalement, la diminution de la consommation était évaluée à - $73 \%$ pour les bourgs de moins de 2000 habitants, $64 \%$ pour les villes de 2000 à 20000 habitants et $-52 \%$ pour celles de 20 à 40000 habitants ${ }^{15}$. La géographie de la consommation ne s'en trouvait pas fondamentalement modifiée mais le produit se trouvait plus ancré dans les consommations des principaux centres urbains, ce qui contribua en partie au renouvellement du marché dans le courant des années 1950.

30 Si la qualité des produits livrés durant toute la période du ravitaillement n'était pas un argument en faveur de leur plus grande diffusion, il n'en reste pas moins que les dynamiques qui se mirent alors en place eurent une influence sensible sur le développement ultérieur du marché et de la consommation. Tout en ayant contribué au renforcement moyen des volumes consommés, l'organisation du ravitaillement contribua à élargir la palette des utilisations des produits, à les penser comme des légumes et à ancrer leur emploi dans les zones urbaines les plus dynamiques en matière de développement de la consommation. Elle joua donc incontestablement un rôle majeur pour la croissance ultérieure de la consommation moyenne.

31 Au sein des industries de biens de consommation, l'industrie des pâtes alimentaires relève bien des branches relativement privilégiées. Son volume d'activité s'est non seulement maintenu, il a même progressé, et les années difficiles furent en fait celles qui suivirent la Libération. L'organisation du ravitaillement permit en outre de consolider la diffusion du produit, dont l'utilisation n'était encore parfois que très épisodique dans certaines régions. Elle contribua à renforcer la consommation dans les grands centres urbains les plus dynamiques. Pour autant, les industriels, à commencer par l'un des premiers d'entre eux, Jean Carret, ne perçurent pas les potentialités d'un élargissement de leurs marchés. Ils tablaient plutôt sur une rentabilisation portée par une réduction de la concurrence et placèrent cet objectif au cœur de leur organisation professionnelle, même si les volontés de restructuration n'eurent alors aucun effet.

Le CPIPA n'en eut pas moins un destin singulier. Maintenu aux fins de la gestion des contingentements qui perdura jusqu'en 1950, il demeura inchangé jusqu'en 1955, date à laquelle des députés s'interrogèrent quant aux motifs de la pérennité d'une institution de Vichy, et ne fut dissous qu'au début des années 1960. Un compromis s'était en fait établi sur son maintien. Les industriels dominants en espérèrent longtemps la mise en œuvre d'une politique volontaire de réduction des capacités de production de la branche tandis que le ministère des Finances y trouvait le moyen efficace du pilotage d'un prix devenu stratégique du fait de son inclusion dans l'indice des prix. En laissant entrevoir une possible réalisation de la politique voulue par les industriels, il incita en fait, sous la pression du contrôle des prix, à de considérables efforts de gains de productivité. Les industriels dominants pouvaient espérer en tirer les dividendes des positions oligopolistiques à venir tout en assurant aux Finances les motifs d'une 
modération des prix. L'organe corporatif fut ainsi un excellent outil de pilotage de la branche.

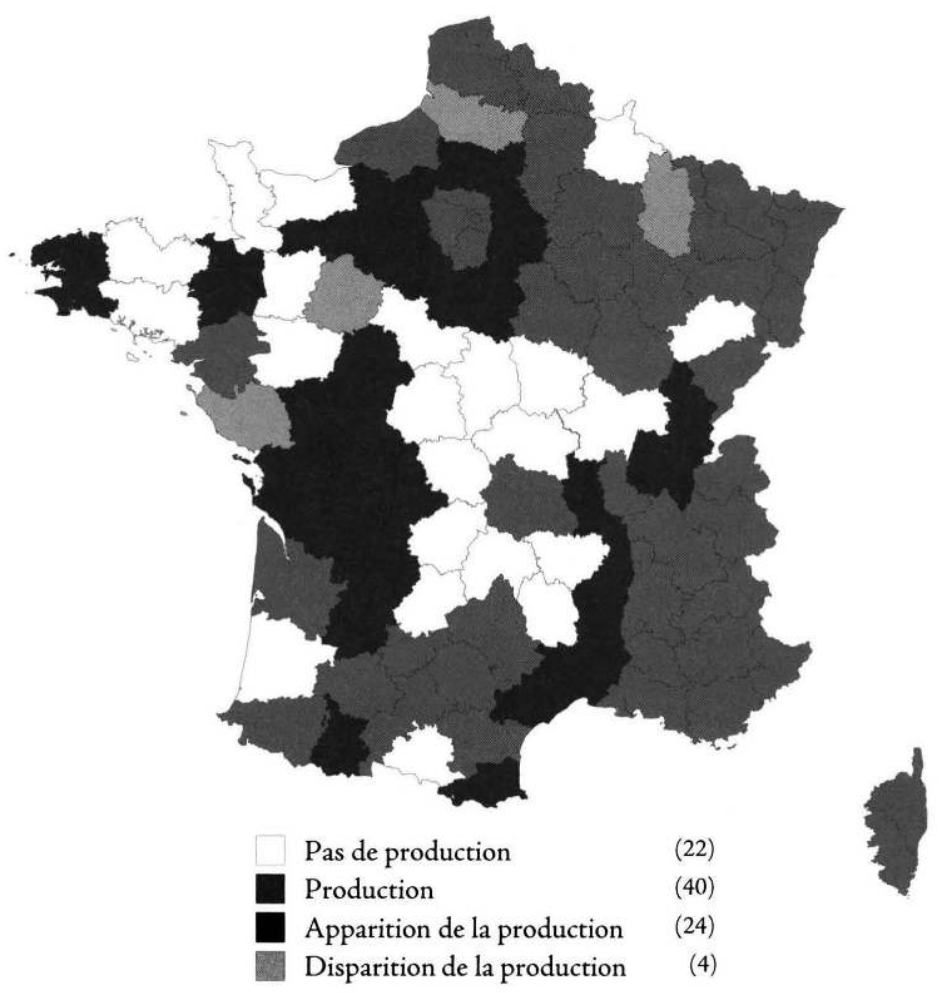

Carte 1. Évolution de la diffusion des établissements en activité entre 1929 et 194816.

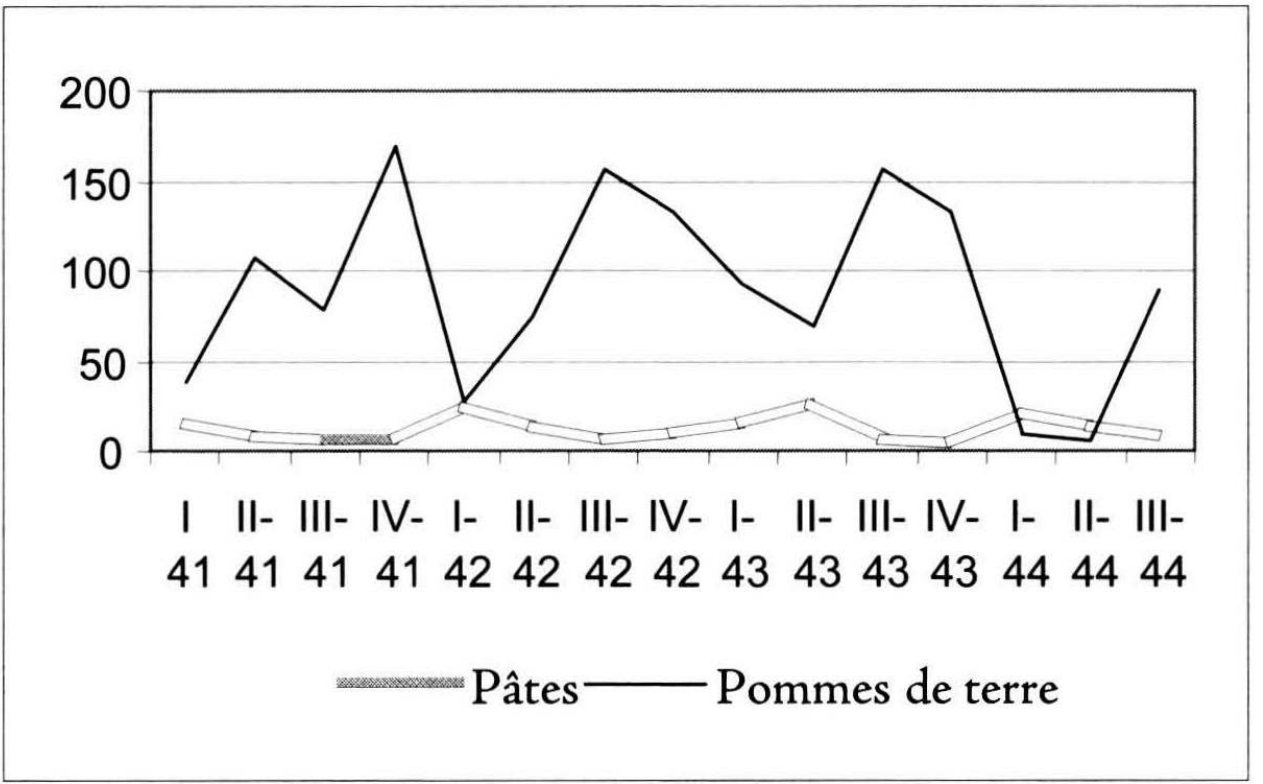

Graphique 1. Rations trimestrielles de pâtes et de pommes de terre 1941-1944 (grammes par jour pour un adulte) $)^{17}$. 


\section{NOTES}

1. Pour une étude détaillée de l'histoire de cette branche, Pierre-Antoine Dessaux, Des vermicelliers au groupe Danone : consommer, produire et vendre des pâtes alimentaires en France, $\mathrm{XVII}^{e}-\mathrm{XX}$ siècles, thèse de doctorat d'histoire (dir. Patrick Fridenson), EHESS, 2003.

2. D'après les données professionnelles, la production serait passée de 148000 tonnes en 1938-1939 à 180000 tonnes en 1939-1940.

3. Documents des archives Rivoire \& Carret (ARC), en possession de l'auteur.

4. ARC, rapport SIPA 1939-1940, s.d., probablement novembre 1940.

5. Journal officiel de l'État français, 26 juin 1941, p. 2691.

6. Ibid., 5 août 1941, p. 3271.

7. Arrêté du ministre secrétaire d'État à l'Agriculture, du ministre secrétaire d'État à l'Intérieur et du secrétaire d'État au Ravitaillement, ibid., 4 février 1942, p. 503.

8. ARC, rapport SIP A, 5 mai 1942.

9. Ibid., 1939/1940.

10. Ibid., 5 mai 1942.

11. Ibid., juin 1944.

12. Pour une étude plus fouillée, Pierre-Antoine Dessaux, «Entrepreneur de la pénurie: la formation d'une entreprise durant les années quarante », dans Jacques Marseille (dir.), Créateurs et créations d'entreprise. De la révolution industrielle à nos jours, Paris, ADHE, 2000, p. 555-578.

13. Michel Chaumet, «Les Deux-Sèvres », dans Dominique Veillon et Jean-Marie Flonneau (dir.), Le Temps des restrictions en France, Cahiers de l'IHTP, n 32-33, 1996.

14. L. Raudoin, J. Maillard, «Les restrictions alimentaires en 1942-1943», Bulletin de la société scientifique d'hygiène alimentaire et d'alimentation rationnelle, Xxxv, 10-11-12,1947.

15. Service des archives économiques et financières, ministère de l'Économie et des Finances, $B$ 57658, inspection générale de l'Économie nationale, direction des Prix, « Étude sur l'industrie des pâtes alimentaires ", 29 mars 1949.

16. Source: Recensement de 1929 et enquête professionnelle menée à partir des années 1940, données publiées dans Pâtes alimentaires, semoules et industries annexes, n 35, 1955.

17. Source : L. Raudoin, J. Maillard, «Les restrictions alimentaires en 1942-1943 », Bulletin de la société scientifique d'hygiène alimentaire et d'alimentation rationnelle, xxxv, 10-11-12,1947.

\section{AUTEUR}

PIERRE-ANTOINE DESSAUX

EHESS, CRH 